\title{
Bioestadigrafía y Análisis Clínico Comparado de 20.000 Casos Obstétricos
}

\author{
Clínica Primero de Mayo ne los Seguros Sociales Colombianos \\ Tesis Doctoral Meritoria (Resumen) - Universidad Nacional, 1953
}

\author{
Por el doctor José Antonio Carvajal-Barrera \\ Médico Residente de la Clínica Primero de Mayo.
}

\section{SUMARIO}

Se revisan las primeras 20.000 hospitalizaciones de la Clínica Primero de Mayo y se obtienen algunos índices estadísticos. Las Clínica inició sus servicios el 10 de junio de 1950; las 20.000 hospitalizaciones se completaron el 11 de mayo de 1953.

Se elabora para cada año el histograma de las estancias utilizadas por las pacientes y se establece el promedio de estancias utilizadas por cada paciente.

Los porcentajes de los cuatro años estudiados son sensiblemente iguales entre sí. Al final se totalizan todos los años y se establecen algunas comparaciones con índices de autores tanto nacionales como extranjeros; se hacen comentarios.

En una segunda parte se resumen en forma de cuadros 497 historias clínicas tomadas al azar durante el año de 1952.

En la misma forma se estudia el total de defunciones maternas acaecidas en el período que se estudia. Se analiza la mortalidad materna de causa obstétrica y se compara el índice de mortalidad con el de algunos autores.

Se hacen breves consideraciones sobre la eclampsia, que constituye la causa principal de mortalidad materna.

Las 20.000 pacientes que se hospitalizaron en la Clínica utilizaron 122.912 estancias, siendo el promedio global de permanencia de cada paciente de 6,4 días. No se discriminó el promedio de permanencia en la Clínica de las pacientes según las diferentes causas de ingreso. Se observa que las pacientes que han 
tenido parto espontáneo, sin complicación, pueden salir sin ningún peligro, entre el tercero y el cuarto día las pacientes que han sido sometidas a cesárea, salvo alguna complicación, pueden abandonar el servicio al décimo día del postoperatorio.

Numerosas pacientes viven fuera del municipio de Bogotá; las ambulancias del ICSS las traen en el momento oportuno. Nos ha parecido que para un scrvicio más eficaz dado el gran movimiento de pacientes, la Clínica debe dis poner para su exclusivo servicio de una o dos ambulancias.

$\begin{array}{ccc}\text { Afiliadas } \ldots \ldots \ldots \ldots \ldots \ldots \ldots \ldots \ldots \ldots \ldots \ldots \ldots \ldots & \begin{array}{r}\text { Casos } \\ 7.706\end{array} & \begin{array}{c}\text { Porcentaje } \\ 38,53 \%\end{array} \\ \text { Beneficiarias } \ldots \ldots \ldots \ldots \ldots \ldots \ldots & 12.294 \\ \text { Total } \ldots \ldots \ldots \ldots \ldots \ldots \ldots & 20.000\end{array}$

Sc denomina beneficiaria a la esposa o compañera del trabajador, que no devenga sueldo alguno y que teóricamente permanece en su casa dedicada a los scrvicios domésticos.

DISCRIMINACION POR CAUSAS DE INGRESO:

\begin{tabular}{|c|c|c|}
\hline & Casos & Porcentaje \\
\hline Por Parto & 16.201 & $81,00 \%$ \\
\hline Por falso trabajo & 917 & $4,59 \%$ \\
\hline Por aborto & 1.441 & $7,21 \%$ \\
\hline Por amenaza de aborto & 570 & $2,85 \%$ \\
\hline Por complicación del embarazo ..... & 531 & $2,66 \%$ \\
\hline En post-partum $\ldots \ldots \ldots \ldots \ldots \ldots$ & 135 & $0,67 \%$ \\
\hline En observación $\ldots \ldots \ldots \ldots \ldots \ldots$ & 181 & $0,90 \%$ \\
\hline Por otras causas (nada obstétrico)... & 24 & $0,12 \%$ \\
\hline
\end{tabular}

Total

20.000

Capasso $^{1}$ en 2.780 partos en la Clínica Primero de Mayo, encuentra 214 abortos, lo que equivale a un $7,6 \%$. Cita Capasso a Simons quien trae un aborto por cada 1,5 partos y a Taussing quien trae un aborto por cada 2,5.

El ICSS no presta sus servicios a la embarazada en quien se han practicado maniobras abortivas criminales.

PARTOS:

\begin{tabular}{|c|c|c|}
\hline & Casos & Porcentaje \\
\hline Unicos & 16.081 & $99,26 \%$ \\
\hline Gemelares & 117 & $0,72 \%$ \\
\hline Trigemelares & 3 & $0,02 \%$ \\
\hline
\end{tabular}

Total

16.201

Se encuentra un parto gemelar por cada 138,4 partos y un parto trigeme lar por cada 5.400,3, aproximadamente. 
Zerda-Jiménez en el Instituto de Protección Materno-Infantil de Bogotá encuentra un parto gemelar por cada 96,09 partos y uno trigemelar por cada 8.581 partos. Veit da una frecuencia del 1,1\% para los partos gemelares y del $0,01 \%$ para los trigemelares.

ANALISIS DE LOS PARTOS:

\begin{tabular}{|c|c|c|}
\hline & Casos & Porcentaje \\
\hline Partos espontáneos & 14.080 & $86,91 \%$ \\
\hline Forceps $\ldots \ldots \ldots$ & 1.585 & $9,79 \%$ \\
\hline$\ldots \ldots \ldots$ & 511 & $3,15 \%$ \\
\hline Versiones internas $\ldots \ldots \ldots \ldots \ldots \ldots$ & 24 & $0,15 \%$ \\
\hline Basiotripsias $\ldots \ldots \ldots \ldots \ldots \ldots$ & 1 & $0,006 \%$ \\
\hline Embriotomías $\ldots \ldots \ldots \ldots \ldots \ldots \ldots$ & 0 & $0,00 \%$ \\
\hline
\end{tabular}

Total

16.201

Corral-Maldonado (1947 y 1948) en el Instituto de Protección Matemo Infantil en 3.130 partos, encuentra un porcentaje del $88,01 \%$ para el parto espontáneo.

Para la aplicación de forceps da Corral-Maldonado una frecuencia del $7,41 \%$, y Capasso para la misma intervención da una frecuencia de $13,27 \%$.

La técnica usada en la cesárea fue la segmentaria transperitoneal con incisión mediana infraumbilical de la piel e incisión transversal del segmento uterino. La anestesia usada fue el ciclo-O2 en buen número de casos, a veces con inducción con pentotal sódico al 2,5\%; en pocos casos se us.j el éter-O2; y en buen número se usó la raquianestesia con scurocaína.

Corral-Maldonado encuentra para la cesárea una frecuencia de 3,41\%. Echeverri-Parra $^{6}$ anota una frecuencia de 1,99\% (1938-1943). Martínez-Sáenz ${ }^{7}$ presenta una frecuencia de $3,1 \%$.

Martínez-Sáenz trae un promedio de frecuencia para la cesárea en los hospitales americanos de 4,9\%. En comparación con este porcentaje, la frecuencia de la operación cesárea entre nosotros, es menor casi en un $2 \%$.

Entre las instituciones nacionales cita Martínez-Sáenz estos clatos referentes a la cesárea:

Instituto de Protección Materno-Infantil de Bogotá, 3,2\%.

Clínica de Marly de Bogotá, 4,8\%.

Hospital San José de Bogotá, 5,9\%.

En las cesáreas practicadas en la Clínica Primero de Mayo no se tuvo en cuenta si el caso era puro o impuro. "Es criterio nuestro no rechazar nunca la intervención laparotómica por el tan cacarcado peligro de inf́ección en los casos llamados sucios" 11.

En 16.201 partos, 24 se terminaron por versión interna, lo que da para esta intervención una frecuencia del $0,15 \%$.

En 16.201 partos ocurridos en la Clínica Primero de Mayo, se practicó una basiotripsia y ninguna embriotomía. 
La embriotomía tiende a facilitarse hoy en día usando para su ejecución instrumentos distintos del gancho de Braun y las tijeras de Dubois, como el embriótomo de Turpin que ha sido dado a conocer y está popularizando entre nosotros Riaño-Alvarez ${ }^{12}$; este embriótomo se asemeja, a grandes rasgos, a un amigdalótomo de asa; en dos demostraciones hechas por Riaño-Alvarez en la Clínica, cl éxito fue completo.

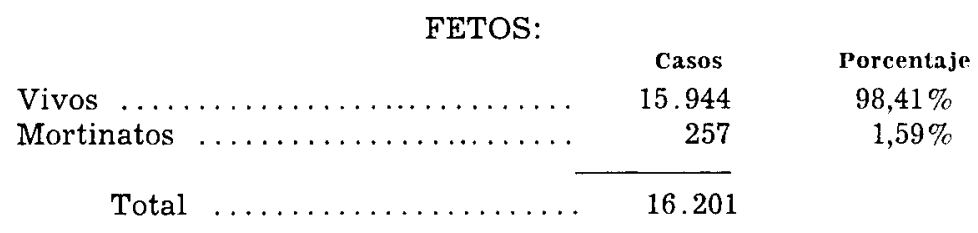

Greenhill trae un índice de mortinatalidad $\in \mathrm{n}$ los Estados Unidos de 21.1 por mil nacimientos, en 1947. En la Clínica Primero de Mayo este índice es de 15,9 por mil nacimientos $(1,59 \%)$.

Kime y Ringer en el Margarett Hague en 1950, anota una frecuencia de mortinatalidad de $1,29 \%$.

Como vemos nuestro porcentaje de mortinatalidad es sensiblemente igual al que traen los autores estadinenses citados. Lesmes-Alford encuentra en el Instituto de Protección Materno-Infantil de Bogotá, en el año de 1944 una frecuencia de mortinatalidad del $5 \%$ aproximadamente.

\section{II}

Esta segunda parte contiene un estudio sobre una serie de 497 historias clínicas, correspondientes a pacientes hospitalizadas del 24 de agosto al 17 de septiembre de 1952.

\begin{tabular}{|c|c|c|}
\hline & Casos & Porcentaje \\
\hline Afiliadas & 198 & $39,84 \%$ \\
\hline Beneficiarias & 299 & $60,16 \%$ \\
\hline
\end{tabular}

Total $\ldots \ldots \ldots \ldots \ldots \ldots \ldots \ldots, 497$

DISCRIMINACION POR EDADES:

\begin{tabular}{|c|c|c|}
\hline & Casos & Porcentaje \\
\hline De 14 años & 1 & $0,20 \%$ \\
\hline De 16 a 25 años $\ldots \ldots \ldots \ldots \ldots$ & 249 & $50,10 \%$ \\
\hline De 26 a 35 años . . . . . . . . . . . . & 163 & $32,80 \%$ \\
\hline De 36 a 45 años & 40 & $8,05 \%$ \\
\hline Sin dato de edad & 44 & $8,85 \%$ \\
\hline Total & 497 & \\
\hline
\end{tabular}


DISCRIMINACION POR EL NUMERO DE EMBARAZOS:

\begin{tabular}{|c|c|c|}
\hline & Casos & Porcentaje \\
\hline Primigestantes & 126 & $25,35 \%$ \\
\hline Secundigestantes $\ldots \ldots \ldots \ldots \ldots \ldots$ & 101 & $20,32 \%$ \\
\hline Tercigestantes .... & 79 & $15,90 \%$ \\
\hline Cuartigestantes & 60 & $12,07 \%$ \\
\hline Multigestantes (cinco o más). & 119 & $23,94 \%$ \\
\hline No embarazadas .. & 12 & $2,42 \%$ \\
\hline Total & 497 & \\
\hline
\end{tabular}

DISCRIMINACION POR CAUSAS DE INGRESO:

\begin{tabular}{|c|c|c|}
\hline & Casos & Porcentaje \\
\hline Por parto & 412 & $82,90 \%$ \\
\hline Por falso trabajo $\ldots \ldots \ldots \ldots \ldots \ldots$ & 10 & $2,01 \%$ \\
\hline Por amenaza de parto prematuro & 2 & $0,40 \%$ \\
\hline Por aborto $\ldots \ldots \ldots \ldots \ldots \ldots \ldots$ & 42 & $8,45 \%$ \\
\hline Por amenaza de aborto $\ldots . \ldots \ldots \ldots$ & 8 & $1,61 \%$ \\
\hline En observación $\ldots \ldots \ldots \ldots \ldots \ldots$ & 10 & $2,01 \%$ \\
\hline Por otras causas (nada obstétrico). . & 13 & $2,62 \%$ \\
\hline \multirow[t]{3}{*}{ Total } & 497 & \\
\hline & & \\
\hline & Casos & Porcentaje \\
\hline Unicos & 407 & $98,79 \%$ \\
\hline Gemelares & 5 & $1,21 \%$ \\
\hline Total & 412 & \\
\hline
\end{tabular}

Det a l l e :

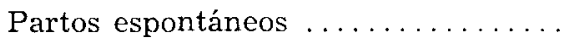

$\begin{array}{rc}\text { Casos } & \text { Porcentaje } \\ 256 & 62,13 \% \\ 41 & 9,95 \% \\ 7 & 1,70 \% \\ 2 & 0,49 \% \\ 49 & 11,89 \% \\ 57 & 13,35 \%\end{array}$

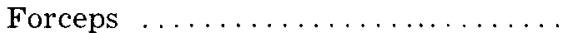

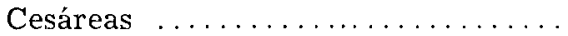

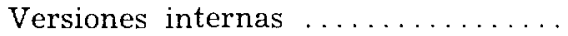

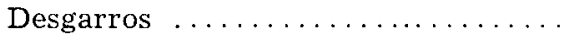

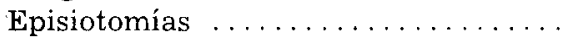

Total

Presentatio nes:

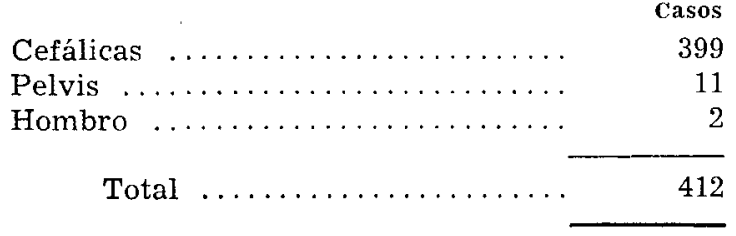

Porcentaje $96,84 \%$ $2,67 \%$ $0,49 \%$ 


\begin{tabular}{|c|c|c|}
\hline \multicolumn{3}{|l|}{ FETOS (417): } \\
\hline & Casos & Porcentaje \\
\hline Vivos & 408 & $97,84 \%$ \\
\hline Mortinatos $\ldots \ldots \ldots \ldots \ldots \ldots \ldots$ & 9 & $2,16 \%$ \\
\hline Total & 417 & , \\
\hline Masculinos $\ldots . \ldots$ & 222 & $53,24 \%$ \\
\hline Femeninos $\ldots \ldots \ldots \ldots \ldots \ldots \ldots$ & 195 & $46,76 \%$ \\
\hline Total & 417 & \\
\hline A término $\ldots \ldots \ldots$ & 350 & $83,93 \%$ \\
\hline Prematuros $\ldots \ldots \ldots \ldots \ldots \ldots$ & 67 & $16,07 \%$ \\
\hline Total ..... & 417 & \\
\hline
\end{tabular}

Bástenos sólo señalar que encontramos 67 prematuros sobre un total de 417 fetos, lo que nos dio una frecuencia del $16 \%$ para los niños prematuros.

Rueda-Vesga después de estudiar las historias de 2.468 partos encontró un porcentaje de prematuros igual al 13,29\%, en el servicio de prematuros de la Clínica Primero de Mayo ${ }^{14}$.

\section{III}

\section{Mortalidad Materna}

En 20.000 hospitalizaciones hubo 31 defunciones, de las cuales solamente 23 fueron de causa obstétrica. Según esto, el índice de mortalidad materna es del $0,11 \%$. Teniendo en cuenta el total de partos ocurridos, este índice de mortalidad sube al $0,14 \%$ o sea 1,4 por mil ( 23 defunciones y 16.201 partos).

Jas otras 8 defunciones se debieron a: TBC; vólvulos intestinal; cardio patía descompensada; ileus intestinal post-operatorio; hemorragia cerebral; hemorragia meníngea; síncope anestésico e infarto del miocardio.

Las causas de las 23 defunciones se discriminan así:

\begin{tabular}{|c|c|c|}
\hline & Casos & Porcentaje \\
\hline Eclampsia & 13 & $56,53 \%$ \\
\hline Hemorragia & 4 & $17,39 \%$ \\
\hline "Shock" obstétrico. & 2 & $8,69 \%$ \\
\hline Aborto séptico ..... & 2 & $8,69 \%$ \\
\hline "Shock" obstétrico-quirúrgico ...... & 1 & $4,35 \%$ \\
\hline Glomerulonefritis ............. & 1 & $4,35 \%$ \\
\hline Total & 23 & \\
\hline
\end{tabular}

Greenhill anota que la mortalidad materna decreció en los Estados Unidos desde 1,6 por mil nacimientos, en 1946, hasta 1,3 por mil en 1947. Las causas principales de mortalidad materna fueron: infección puerperal ( $32 \%$ del total); hemorragia, trauma y schock (31\%); toxemia (27\%). 
Según Dickinson y Welker ${ }^{15}$ el dato general para los Estados Unidos en 1949 es el de una muerte $(0,98 \%)$ por mil nacidos vivos. En 1948 la principal causa de muerte estuvo constituída por las toxemias del embarazo y del puerperio, con un $26,7 \%$ del total de las defunciones.

Traen estos mismos autores los siguientes índices de mortalidad materna de las naciones que lo tienen más bajo:

\begin{tabular}{|c|c|c|c|}
\hline Australia (excluyendo aborigenes) en $1947 \ldots$ & 1,9 & por & mil. \\
\hline$\ldots \ldots \ldots \ldots \ldots \ldots \ldots \ldots \ldots$ & 1,5 & ", & , \\
\hline Dinamarca en 1948 & 0,9 & ", & \\
\hline Inglaterra en 1947 & 1,2 & $"$ & \\
\hline Finlandia en 1947 . & 2,1 & $"$ & , \\
\hline Países Bajos en $1947 \ldots \ldots$ & 1,4 & $"$ & " \\
\hline Noruega en $1947 \ldots \ldots$. & 1,5 & $"$ & , \\
\hline Suecia en 1945 . & 1,3 & " & $"$ \\
\hline Suiza en 1948 & 1,8 & $"$ & $"$ \\
\hline
\end{tabular}

De acuerdo con los autores estadinenses que acabamos de mencionar, vemos que el índice de mortalidad materna en la Clínica Primero de Mayo, está muy cerca del existente en los Estados Unidos. Este índice es más halagador si tenemos en cuenta que algunas de las pacientes fallecidas, especialmente cclámpticas, ingresaron a la Clínica en estado preagónico y murieron pocas ho. ras después de su ingreso.

Queda consignado pues que la eclampsia produjo el $56,53 \%$ de los decesos.

Greenhill anota que la mortalidad materna por eclampsia es del $13 \% \mathrm{cn}$ los hospitales de los Estados Unidos.

Gordon, Rosenthal y Oleary ${ }^{17}$ anotan que en la eclampsia se encuentra el más fértil campo para una amplia reducción de la mortalidad materna.

Hamlin $^{18}$ dice que la eclampsia puede abolirse; esto se consigue con un adecuado cuidado prenatal.

Fitzgerald y Webster ${ }^{16}$ concluyen: "La mayor esperanza para una amplia reducción de la mortalidad materna descansa en la completa eliminación de las muertes debidas a toxemia. Esto se puede realizar con un adecuado cuidado prenatal y con un mayor convencimiento por parte de los médicos de que la mortalidad por toxemia es prácticamente cero si se evita que la paciente llegue al periodo de las convulsiones eclámpticas".

De acuerdo con estos conceptos y teniendo en cuenta las numerosas muer. tes debidas a la eclampsia, podemos decir que en nuestro medio se puede reducir la mortalidad materna si se propende porque sean menos frecuentes los casos de eclampsia que llegan a la Clínica. Para lograr esto, se deben tomar diversas medidas tendientes a una mejor asistencia prenatal, ya que la toxemia en su principio se controla más con medidas de higiene del embarazo que con tratamiento medicamentoso.

La hemorragia fue después de la eclampsia, la principal causa de defunción en la Clínica Primero de Mayo (17,39\%). Contra la hemorragia sólo podemos luchar con éxito mediante la oportuna transfusión sanguínea, para lo cual el Banco de Sangre debe encontrarse instalado en el servicio hospitalario. 


\section{CONCLUISIONES}

19-Del 10 de junio al 31 de diciembre de 1950 ingresaron a la Clínica 3.249 pacientes, que utilizaron 22.324 estancias. En promedio cada paciente permaneció hospitalizada durante 6,8 días.

$2^{\circ}$-En el año de 1951 se hospitalizaron 6.735 pacientes, que produjeron +4.478 estancias. Cada paciente permaneció en promedio 6,6 días.

30.-Fn 1952 se hospitalizaron 7.242 pacientes, que utilizaron 40.870 estancias. En promedio cada paciente permaneció en la Clínica 5,6 días.

49-Entre el $1^{\circ}$ de enero y el 11 de mayo de 1953 se hospitalizaron 2.774 pacientes, las cuales utilizaron 15.240 estancias. Cada paciente permaneció en la Clínica 5,4 días en promedio.

50--Más de la mitad (61\%) de las pacientes que reciben atención en la Clínica Primero de Mayo son beneficiarias.

$6^{\circ}$-La gran mayoría de las pacientes ingresadas lo fueron por parto $(81 \%)$; siguen como causa de ingreso el aborto (7\%), el falso trabajo (5\%), la amenaza de aborto y la complicación del embarazo ( $3 \%$ cada una).

$7^{\circ}$-La frecuencia de los partos múltiples, gemelares y trigemelares, es prácticamente igual a la que dan la mayoría de los tratadistas.

8.-La gran mayoría de los partos se produjeron de manera espontánea $(86,91 \%)$ y un $9,79 \%$ de los partos se terminaron por medio del forceps.

90-La cesárea se practicó en la Clínica Primero de Mayo con una frecuencia de $3,15 \%$, frecuencia menor a la que se encuentra en los principales hospitales norteamericanos y a la que arrojan el servicio de pensionados del Hospital de San José y de la Clínica de Marly.

$10^{\circ}$-Encontramos para la versión interna una frecuencia del $0,15 \%$ considerablemente menor que la encontrada por Corral-Maldonado en el Instituto de Protección Materno-Infantil.

11 - - Se practicó una basiotripsia en 16.201 partos; no hubo embriotomías. La incidencia de estas intervenciones es notoriamente menor a la que se encuentra en el Instituto de Protección Materno-Infantil.

$12^{\circ}$ - La incidencia de fetos mortinatos $(1,59 \%)$ es sensiblemente igual a la que traen los autores norteamericanos $(2,1 \%$ y $1,29 \%)$ y bastante menor de la que se encuentra en el Instituto de Protección Materno-Infantil.

13ㅇ-El bajo índice de mortalidad materna de la Clínica Primero de Mayo ( 1,4 por mil) es prácticamente igual al que arrojan los mejores centros hospitalarios de los Estados Unidos v está muy cerca del índice de las naciones que lo tienen más bajo. Este índice habla de la eficiencia de los servicios que se prestan en la Clínica Primero de Mayo.

140-La colampsia fue la principal causa de mortalidad materna en la Clí nica (56,53\%); sigue en orden de frecuencia la hemorragia (17,39\%), cl shock obstétrico y el aborto séptico (8,69\% cada uno). En los Estados LInidos las principales causas de defunción materna son las toxemias, la infección y la hemorragia.

150-El alto porcentaje de muertes maternas producidas por la cclampsia 
puede reducirse considerablemente. Para lograrlo se deben tomar las medidas necesarias para una mejor asistencia prenatal y una mayor concurrencia por parte de las cmbarazadas a los consultorios o dispensarios prenatales.

$16^{\circ}$-Es aconsejable que en la Clínica se instale un banco de sangre, para que sin pérdida de tiempo se pueda disponer de este vital elemento en el momento oportuno.

\section{BIBLIOGRAFIA}

1.-Capasso Camilo. "Consideraciones sobre 3.000 partos". Revista Colombiana de Obstetricia y Ginecología, Vol. III, No. 3: 108-114, y No. 4: 154.

2.-Cartoux G. et Tran-dinh-de. "Sur une serie de 301 grossesses extra-utérines". Gynécologie et Obstétrique, París, Tome 49, No. 4: 390, 1950.

3.-Zerda-Jiménez Leopoldo. "Anotaciones clínico-estadísticas sobre el embarazo y parto múltiples". Tesis de grado, Universidad Nacional, 1948.

4.-Greenhill J. P. "Principles and practice of obstetrics". Tenth Edition, Saunders, Philadelphia, 1951, pág. 435.

5. Corral-Maldonado Jaime. "Las grandes intervenciones obstétricas. Estudio clínico y estadístico en 2 años". Revista Colombiana de Obstetricia y Gine." cología, Vol. II, No. 2: 49-68, y No. 3: 90-112.

6. -Echeverri-Parra Gonzalo. "Consideraciones clínicas y estadísticas sobre operación cesárea". Tesis de grado, Universidad Nacional, 1944.

7.-Martínez-Sáenz Carlos. "Operación cesárea en la Clínica Primero de Mayo". Inédito, 1951. Trabajo leído el 10 de septiembre de 1953 en la Primera Convención Nacional de Obstetricia y Ginecología reunida en Bogotá.

8.-Kinne R. and Ringer N. "Margaret Hague Maternity Hospital. Cesarean section report. 1950". October 8, 1951. Publicación en mimeógrafo.

9.-Tran-Dinh-De. "Remarques sur une série de 282 cas de césarienne abdominale". Gynécologie et Obstétrique, Paris, Tome 49, No. 4: 400-404, 1950.

10.-Hugdieter Noack. "Zur "Krise" der Kaiserschnitt-Indikation". GeburthFrauenh, 1952, 12: 104. Extractado en Surg. Gynec. and Obst., 95: 570, December, 1952.

11.-Mascaro-Porcar José María. "Breviario de la Urgencia Obstétrica". Segunda Edición, Salvat Editores, S. A., Barcelona-Buenos Aires, 1945. Págs. 79 y 80.

12.-Riaño Alvarez Gustavo. "Embriotomía Raquídea". Trabajo inédito presentado a la Primera Convención Nacional de Obstetricia y Ginecología de Colombia y leído en la sesión del 10 de septiembre de 1953.

13. - Lesmes-Alford Guillermo. "Mortinatalidad y Mortalidad precoz. Instituto de Protección Materno-Infantil". Tesis de grado, Universidad Nacional, 1949.

14.- Rueda-Vesga Custodio. "Atención hospitalaria del prematuro". Tesis de grado. Universidad Nacional, 1952.

15.-Dickinson Frank G. and Welker Everet L. "Maternal Mortality in the United States en 1949". J. A. M. A., 144: 1.395-1.400, December, 1950.

16.-Fitzgerald J. E. and Webster Augusta. "Nineteen-year survey of maternal mortality at the Ccok Counto Hospital". Am. J. Obst. and Gynec., 65: 528-538, March, 1953.

17. Gordon Charle A., Rosenthal A. H. and Oleary J. L. "Eclampsia and Preeclampsia", Am. J. Surg., 81: 645-654, June, 1951.

18.-Hamlin R. H. J. "The prevention of eclampsia and pre-eclampsia". Lancet, Lond., 1952, 262: 64. Extractado en Surg. Gynec. and Obst., 94: 559, June, 1952.

N. de la R.: La Clínica Primero de Mayo está atendida por obstetras de la Sociedad Colombiana de Obstetricia y Ginecología. 